\title{
Characterization of DC Current Sensors With AC Distortion for Railway Applications
}

\author{
Helko E. van den Brom ${ }^{\circledR}$, Senior Member, IEEE, Ronald van Leeuwen, and Ralph Hornecker
}

\begin{abstract}
To assess the reliability of dc energy measurement equipment on-board trains, a setup was developed to characterize current transducers under realistic operating conditions. The operating principle is based on a current ratio measurement technique. The reference sensor is a high-precision zero-flux current transducer in combination with a broadband highprecision current shunt. The influence of ac distortion on this reference sensor was found to be within a few parts in $10^{6}$ using an initial version of the setup, in which ac distortion was applied through a separate winding. A revised version of the setup employs a programmable electronic load to apply dynamic currents up to $600 \mathrm{~A}$. The setup was used to characterize a $100 \mu \Omega$ high-current shunt resistor. The effect of dissipative heating on the dc transresistance error was around $0.03 \%$, with a settling time of about half an hour. The short-term intrinsic current dependence was also around $0.03 \%$. The effect of ac distortion was within a few parts in $10^{6}$. The intrinsic current dependence and the onset of the heating effect were also observed when exposing the sensor to a dynamic current profile that was recorded during a trip between two successive underground train stations on Metro de Madrid. These results demonstrate that the setup described in this paper is very effective for characterizing dc current sensors for practical railway applications. Future work will concentrate on even more demanding current signals, such as chopped signals, and on other types of sensors and measurement systems.
\end{abstract}

Index Terms-Current measurement, current sensors, current transducers, measurement standards, measurement techniques, measurement uncertainty, precision measurements.

\section{INTRODUCTION}

$\mathbf{M}$ ARKET liberalization in railway transportation and the European Union target of $50 \%$ reduction in $\mathrm{CO}_{2}$ emission by 2030 have set the need to accurately measure the energy consumption of individual trains. In railway systems, energy reduction can be realized by different means, such as regenerative braking, energy-efficient driving, reduction in traction losses, optimization of comfort functions, energy metering, smart power management, and renewable energy microgeneration [1]. In all cases, accurate and reliable mea-

Manuscript received July 7, 2018; revised November 12, 2018; accepted January 25, 2019. Date of publication March 6, 2019; date of current version May 10, 2019. The research leading to the results described in this paper is performed within the 16ENG04 MyRailS Project of the European Metrology Programme for Innovation and Research (EMPIR). The EMPIR initiative is co-funded by the European Union's Horizon 2020 Research and Innovation Programme and the EMPIR participating states, including the Dutch Ministry of Economic Affairs and Climate Policy. The Associate Editor coordinating the review process was Djamel Allal. (Corresponding author: Helko E. van den Brom.)

The authors are with VSL B.V., 2600 AR Delft, The Netherlands (email: hvdbrom@vsl.nl).

Digital Object Identifier 10.1109/TIM.2019.2898014 surement of the amount of consumed, regenerated or stored energy is essential to quantify the actual reduction obtained.

European legislation requires that energy billing is based on the measurement equipment on-board trains by 2019 [2]. However, due to harsh operating conditions, such as arcing, caused by nonideal pantograph to overhead line contacts, extreme temperatures, rapid acceleration, and deceleration of the train, accurately measuring the current on-board trains is challenging. These harsh conditions create all kinds of electrical distortion influencing the measurement of electrical parameters on-board trains.

The so-called energy measurement function (EMF) that should be implemented on-board trains provides the measurement of the consumed and regenerated energy of a traction unit, as set out in [3]. The EMF consists of a voltage measurement function, a current measurement function, and an energy calculation function. The voltage and current measurement functions include voltage and current transducers, respectively, as well as data-acquisition units. This paper focuses on the current measurement function for dc traction supply systems, which measures the dc current taken from and returned to the contact line system.

To verify and test the current measuring equipment under realistic operating conditions as described earlier, a traceable measurement setup is developed to calibrate and characterize dc current sensors or complete dc current measurement chains, under adjustable nonideal conditions. Using this setup, nonideal operating conditions can be simulated not only for railway applications but also for other electrical systems used in transportation, such as for aircraft, ship propulsion, or road vehicles.

Literature shows that monitoring of dynamic and highly distorted signals for ac or dc high-current applications has been investigated earlier (see [4]-[7]). Nevertheless, no reference setup has been developed to provide traceability to international measurement standards for calibrations of the equipment used to measure such dynamic and distorted signals. Until now, traceable calibration systems for high-current measurement systems have only been presented for static signals (see [8], [9]). For example, work on a testbed similar to the system described in this paper, which has been performed in parallel recently [6], does not show traceability of the reference current sensor under distorted conditions.

In previous work [10], a setup was described comparing two transducers measuring the same dc current, whereas one was exposed to a superimposed ac ripple distortion as well. In this paper, we explain how the results are used to 
extend the setup to include more general nonideal signals. The operating principle of the setup is explained, and its capabilities are demonstrated. The measurement results are presented characterizing a dc high-current shunt by means of direct comparison to a high-precision reference sensor.

\section{Current Sensors}

\section{A. Types of Current Sensors}

Two types of current sensors can be distinguished. The first type of sensor converts current to voltage, such as a current shunt for dc systems or a Rogowski coil for ac systems. For these types of sensors, the transresistance or transimpedance value is the relevant parameter to be determined, defined as the ratio between the output voltage and the input current for dc or ac systems, respectively.

The second type of sensor converts a high input current to a much lower output current, such as a zero-flux current transducer [11] with current output for dc systems or a current transformer for ac systems. For these types of sensors, the current ratio between input (or primary) current and output (or secondary) current is the relevant parameter to be determined. For these current-to-current transducers, an auxiliary high-precision current shunt can be used to convert the secondary current to voltage to be able to perform accurate measurements or for direct comparison to current-tovoltage sensors. For simplicity, in this paper, the combination of a current-to-current sensor and an auxiliary current shunt will be considered a current-to-voltage sensor.

\section{B. Definition of the Measurand}

In this paper, the error is the deviation from the nominal value of the current ratio or transresistance, expressed as a relative value with respect to the nominal value. For a current-to-current sensor that converts a primary current $I_{\mathrm{p}}$ to a secondary current $I_{\mathrm{s}}$, the current ratio error is defined as

$$
\varepsilon=\frac{n \cdot I_{\mathrm{s}}}{I_{\mathrm{p}}}-1
$$

where $n$ is the nominal current ratio of the current sensor. The transresistance error of a current-to-voltage sensor that converts an input current $I_{\text {in }}$ to an output voltage $V_{\text {out }}$ is defined as

$$
\varepsilon=\frac{V_{\text {out }}}{R_{\text {nom }} \cdot I_{\text {in }}}-1
$$

where $R_{\text {nom }}$ is the nominal transresistance value of the sensor.

In case of a current-to-voltage sensor that is compared to a current-to-current sensor, the same input or primary current $I_{\text {in }}$ or $I_{\mathrm{p}}$ is sent through both sensors in series. Hence, from the definitions (1) and (2), one can derive that the error of the device under test (DUT), $\varepsilon_{\text {dut }}$, can be expressed in terms of the ratio of the two measured secondary currents $I_{\mathrm{s}}^{\text {ref }}$ and $I_{\mathrm{s}}^{\text {dut }}$ or output voltages $V_{\text {out }}^{\text {ref }}$ and $V_{\text {out }}^{\text {dut }}$, the nominal current ratios of the reference sensor and the sensor under test $n_{\text {ref }}$ and $n_{\text {dut }}$ or the nominal transresistance values $R_{\text {nom }}^{\text {ref }}$ and $R_{\text {nom }}^{\text {dut }}$ and the error of the reference sensor, $\varepsilon_{\text {ref }}$. For the situation described in this paper, in which a current-to-voltage sensor is compared to a current-to-current sensor, the corresponding equation reads

$$
\varepsilon_{\mathrm{dut}}=1-n_{\mathrm{ref}} \cdot R_{\mathrm{nom}}^{\mathrm{dut}} \cdot \frac{I_{\mathrm{s}}^{\mathrm{ref}}}{V_{\mathrm{out}}^{\text {dut }}}+\varepsilon_{\mathrm{ref}}
$$

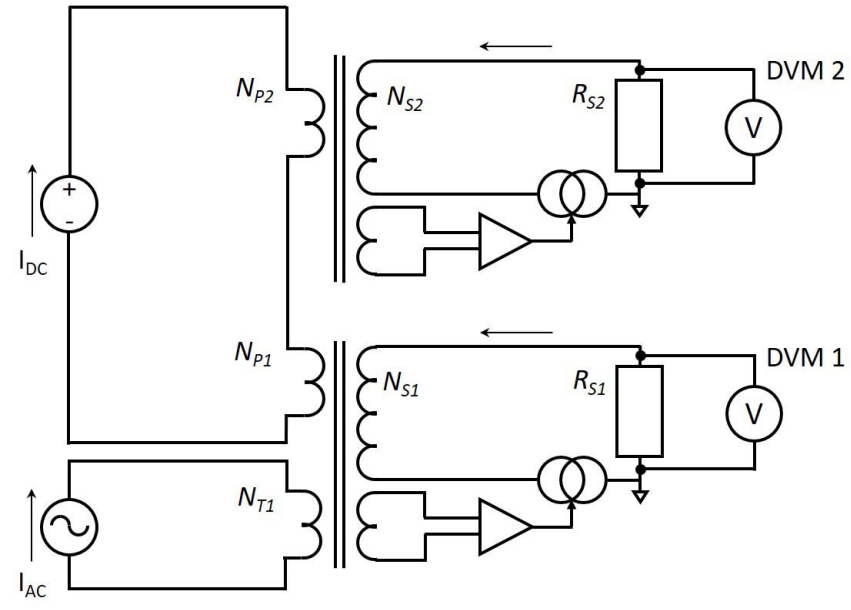

Fig. 1. Schematic of the initial measurement setup to compare two current sensors, labeled 1 and 2 , at a dc current $I_{\mathrm{dc}}$ with ac distortion $I_{\mathrm{ac}}$. Both sensors consist of a zero-flux current transducer, with $N_{\mathrm{P}}$-turns primary winding and $N_{\mathrm{S}}$-turns secondary winding, in combination with an auxiliary broadband high-precision shunt resistor $\left(R_{\mathrm{S}}\right)$. The ac distortion is applied through a separate $N_{\mathrm{T}}$-turns primary winding. The output voltage of both sensors is measured using a high-precision DVM.

where the equality holds up to the first order in the ratio errors. Hence, the transresistance error of the sensor under test can be directly read from the measured ratio of the secondary current and output voltage after a simple correction for the ratio error of the reference sensor. Note that (3) can be rewritten as

$$
\varepsilon_{\mathrm{dut}}=1-\frac{R_{\mathrm{nom}}^{\mathrm{dut}}}{R_{\text {nom }}^{\text {ref }}} \cdot \frac{V_{\text {out }}^{\text {ref }}}{V_{\text {out }}^{\text {dut }}}+\varepsilon_{\text {ref }}
$$

where now the combination of the current-to-current reference sensor and the auxiliary current shunt resistor $R_{\text {aux }}$ is considered a current-to-voltage sensor having a transresistance equal to $R_{\text {nom }}^{\text {ref }}=R_{\text {aux }} / n_{\text {ref }}$.

\section{Measurement Setup for Static Signals}

\section{A. Principle of Operation}

The basis of the measurement setup to characterize dc current sensors under distorted operating conditions lies in a current ratio measurement technique initially developed for comparing ac current transformers [8]. The operating principle of the setup is to compare the sensor under test to a reference sensor that is calibrated using a primary reference setup [9] and, therefore, traceable to international measurement standards. When the two sensors are connected in series to measure the same input current, the ratio of their output voltages can be used to determine the transresistance error of the sensor under test by means of (4). The harsh operating conditions can be mimicked by applying various nonideal test signals.

In the initial version of the setup, only static test signals can be applied, composed of a dc current with or without ac distortion [10]. Furthermore, the sensor under test needs to contain a zero-flux current transducer when the ac distortion is applied. The corresponding schematic is shown in Fig. 1. The dc primary current is generated by means of a $100 \mathrm{~A} \mathrm{dc}$ current source and is applied to the primary winding of the 
two sensors to be compared. The ac distortion is induced using a separate winding through the sensor under test.

\section{B. Sampling Ratio Measurements}

The ratio of the output voltages of the two sensors to be compared is measured using two high-precision digital voltmeters (DVMs), either in dc voltage mode for dc voltages or in sampling mode using an asynchronous sampling technique [12] when measuring ac distorted signals.

For ac distorted signals, the dc component is defined as the zero-frequency component of the Fourier spectrum, which is equal to the average of all samples in the sampling time window. To avoid spectral leakage and, thus, low-frequency ac modulation of the dc component and measurement errors, the signal is resampled using spline interpolation at a rate that is an integer multiple of the repetition rate of the ac distortion waveform.

When using the asynchronous sampling technique, the aperture time of the sampling voltmeters used is set to $100 \mu \mathrm{s}$ with a sampling rate of $8 \mathrm{kS} / \mathrm{s}$, taking 12000 samples in a single reading using a moving average of ten readings. In the dc voltage mode, three measurements are performed per second.

Using asynchronous sampling, the voltage ratio can be determined with an accuracy of better than two parts in $10^{6}$, which is limited by the linearity of the voltmeters [8].

\section{Current Sensors to be Compared}

The sensor under test in this initial setup, labeled 1 in Fig. 1, is in fact meant to be used as a reference sensor in a revised setup as described in chapter $\mathrm{V}$, allowing for different distortion signals and other types of transducers. This sensor is a high-precision zero-flux current transducer with a nominal ratio of 1500:1, a 900 A maximum primary current, and $1 \mathrm{MHz}$ bandwidth, in combination with a broadband $1 \mathrm{~A}$ current shunt to convert the output current to voltage. The dc current ratio of this zero-flux current transducer and the intrinsic current dependence over a wide range of input currents were determined with an accuracy of $1 \mu \mathrm{A} / \mathrm{A}(k=2)$ using a direct current comparator bridge that is originally designed for high-current low-resistance calibrations [9]. The broadband 1 A current shunt was designed to have very low ac-dc difference for frequencies up to $100 \mathrm{kHz}$ [13] and calibrated at dc using standard resistance calibration techniques with an accuracy of better than $1 \mu \Omega / \Omega(k=2)$. The high bandwidth of both zero-flux current transducer and current shunt is necessary to allow for dynamic current signals, as further discussed in Section V.

As a second sensor, labeled 2 in Fig. 1, a similar zero-flux current transducer is used with a nominal ratio of 600:1 and 60 A maximum primary current, in combination with a broadband high-precision 100-mA current shunt. This zero-flux current transducer is stable within the time of measurement but only accurate to the level of $200 \mu \mathrm{V} / \mathrm{V}$. This sensor is used as a stable reference for this measurement to verify the behavior of the first transducer under ac distortion.

The major difference in nominal operating currents of the two transducers is largely compensated by looping the dc primary conductor ten times through the primary winding of
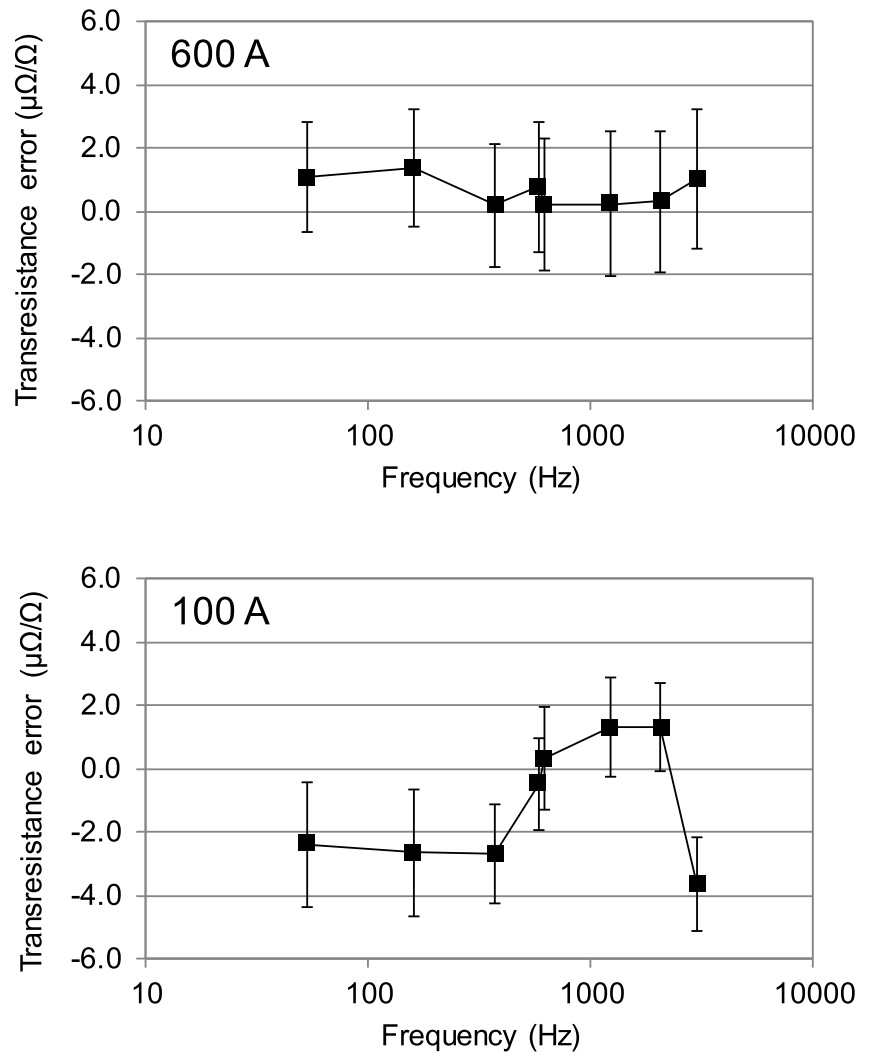

Fig. 2. Relative effect of an ac distortion current on the dc output voltage of the reference transducer for a dc current of 600 A (upper curve) and 100 A (lower curve). The same ac distortion current is applied in the two cases.

the first transducer. DC currents up to $60 \mathrm{~A}$ are then sent through the ten-turn primary winding of the latter transducer (effectively resulting in primary dc currents up to $600 \mathrm{~A}$ ) and through the single-turn winding of the second transducer.

The ac distortion, with effective rms magnitudes up to $24 \mathrm{~A}$, is exclusively applied to the first transducer by means of a separate 24-turn winding. An isolation transformer is used to avoid possible dc offsets in the ac circuit that would not be sensed by the second transducer, thus erroneously changing the ratio of the dc output readings.

\section{The Results of the Setup for Static Signals}

For the setup indicated in Fig. 1, ac distortion signals were applied with rms magnitudes of $24 \mathrm{~A}$ for frequencies up to $1 \mathrm{kHz}$, whereas for higher frequencies, the rms magnitude was decreased to $8 \mathrm{~A}$ at $3 \mathrm{kHz}$ to satisfy the compliance limit of the transconductance amplifier used to generate the distortion current. Measurements were performed at different frequencies for effective dc currents of 600 and $100 \mathrm{~A}$. The same ac distortion current is used in both cases, such that for the dc current of $600 \mathrm{~A}$, the relative level of distortion is six times lower than for 100 A (i.e., $4 \%$ as compared to $24 \%$, respectively).

The transresistance error due to the ac distortion current on the dc output voltage of the reference transducer is presented in Fig. 2. The error bars show the standard deviation of the mean when using the moving average of the individual readings. 
TABLE I

UnCERTAINTy BUdget OF THE REFERENCE SENSOR AT 600 A DC WHEN USED UNDER DisTORTED CONDITIONS

\begin{tabular}{lccc}
\hline $\begin{array}{l}\text { Uncertainty } \\
\text { component }\end{array}$ & $\begin{array}{c}\text { Uncertainty } \\
\text { contribution } \\
(\mu \Omega / \Omega)\end{array}$ & $\begin{array}{c}\mathrm{k}- \\
\text { factor }\end{array}$ & $\begin{array}{c}\text { Standard } \\
\text { uncertainty } \\
(\mu \Omega / \Omega)\end{array}$ \\
\hline \hline Primary standard & 1.0 & 2 & 0.5 \\
$\begin{array}{l}\text { Effect } \\
\text { distortion }\end{array}$ & 5.0 & $\sqrt{ } 3$ & 2.9 \\
$\begin{array}{l}\text { Voltmeter ratio } \\
\text { Standard deviation }\end{array}$ & 2.0 & $\sqrt{ } 3$ & 1.2 \\
\hline Total $(\mathrm{k}=2)$ & 2.5 & 1 & 2.5 \\
\hline
\end{tabular}

When applying a dc current of $600 \mathrm{~A}$, the ac distortion seems to have negligible effect, as can be seen in the upper graph in Fig. 2. When reducing the applied dc current to $100 \mathrm{~A}$ and keeping the same amplitude of the ac distortion, however, a minor effect can be observed in the lower graph, though without a clear trend.

From these observations, it can be concluded that the influence of severe ac distortion on the dc output reading of the reference transducer is within five parts in $10^{6}$.

The total uncertainty of the reference transducer when used under distorted conditions is determined by the dc calibration using the primary reference setup [9], the influence of the ac distortion as described in this section, the accuracy of the voltmeter ratio, and the standard deviation of the measurements (type A uncertainty). The total uncertainty budget for a dc input current of $600 \mathrm{~A}$ is shown in Table $\mathrm{I}$; the total expanded uncertainty is $6.5 \mu \Omega / \Omega(k=2)$. For dc currents down to $100 \mathrm{~A}$, the total uncertainty will be slightly larger but still well within $10 \mu \Omega / \Omega(k=2)$.

\section{Measurement Setup for Dynamic Signals}

\section{A. Principle of Operation}

The idea of the improved setup is to be able to measure current sensors not exclusively based on zero-flux current transducers. At the same time, by means of this setup, one should be able to apply a broader range of test signals. The operating principle and the sampling technique are similar to the initial setup described in Section III.

Fig. 3 shows the schematic of the revised measurement setup. The dc primary current $I_{\mathrm{p}}$, sent through the two sensors to be compared, is generated by means of a 900 A high-power dc current source and modulated using a programmable dc electronic load $Z_{\text {load }}$ that is connected in series. The load can be programmed to simulate various load profiles with unipolar currents up to 600 A with frequency components up to $20 \mathrm{kHz}$. Examples relevant for railway applications are dc current with ac distortion (ripple), dc current varying with time, or userdefined waveforms. In this way, the DUT can be characterized under conditions that resemble normal operation conditions for the current sensor, found in traction units or in other electrical systems in transportation applications.

Note that by adapting the setup, any combination of currentto-current and current-to-voltage sensors can be compared by adding or removing proper auxiliary high-precision shunts. In fact, even complete current measuring systems including

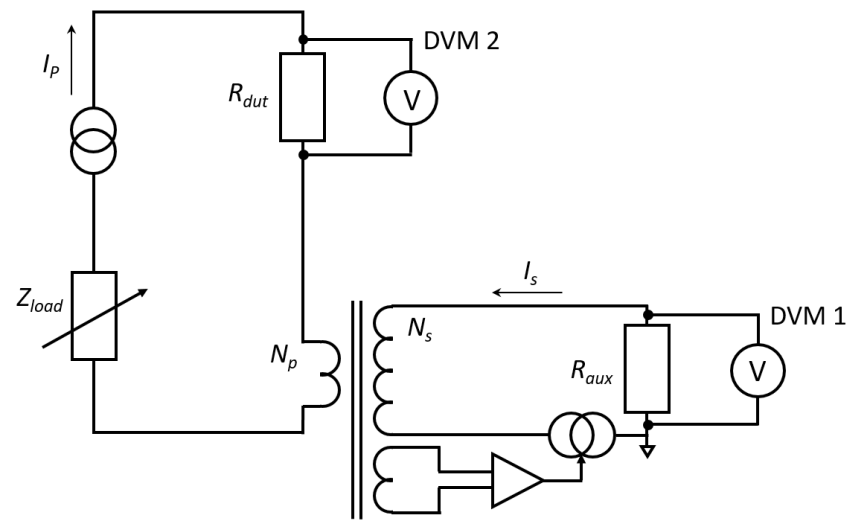

Fig. 3. Schematic of the measurement setup to characterize a dc current sensor using dynamic current signals. The DUT here is a high-current shunt $\left(R_{\text {dut }}\right)$. The reference current sensor consists of a zero-flux current sensor in combination with an auxiliary high-precision shunt resistor $\left(R_{\mathrm{aux}}\right)$.

readout ammeter or voltmeter can be characterized when leaving out the second DVM.

\section{B. Current Sensors to be Compared}

In this paper, the working principle of the revised measurement setup will be demonstrated using the setup shown in Fig. 3. The current sensor under test, in this case, is a high-current shunt with resistance $R_{\text {dut }}$ of nominally $100 \mu \Omega$, designed for operation for currents up to $300 \mathrm{~A}$ and equipped with an active cooling fan.

The reference current sensor in the revised setup is the combined zero-flux current transducer and auxiliary high-precision current shunt $R_{\text {aux }}$ investigated in the previous chapter. As in this paper, the typical currents are a few times smaller than the 900 A maximum input, a two-turn primary winding is used to increase the effective input current to $600 \mathrm{~A}$ when applying $300 \mathrm{~A}$ to the shunt under test, for two reasons, i.e., for demonstrating operation of the setup for currents up to $600 \mathrm{~A}$ and for increasing the sensitivity of the sensor.

\section{Results of the Setup fOR Dynamic Signals}

Various measurements have been performed to characterize the current shunt under test, as described more extensively in a separate publication [14]. These measurements were designed to isolate the influence of the stability of the shunt, intrinsic current dependence, response to dynamic loading, and the effect of ac distortion. Specific tests for other applications, such as using chopped signals, can be performed as well.

To calibrate the transresistance value of the current shunt under test and to investigate the possible effect of self-heating, the transresistance error is measured at a dc current of $270 \mathrm{~A}$. As can be seen in Fig. 4, after settling for about half an hour, the final value is about $0.03 \%$ lower than the value found directly after switching on the current. By investigating the corresponding individual current recordings of the two sensors, it is observed that the drift is caused by the shunt, whereas the reference sensor shows no drift at all, as expected. Note that this effect of self-heating is adjacent to the influence of the external temperature, which is less than five parts in $10^{6}$ per degree Celsius according to the manufacturer's specifications, 


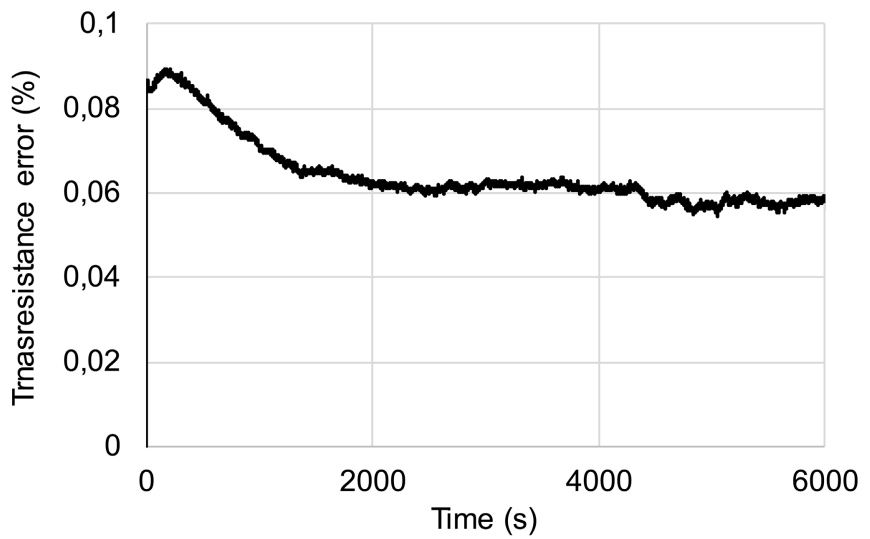

Fig. 4. Measured transresistance error of the current shunt under test as a function of time after applying a static dc current of $270 \mathrm{~A}$.

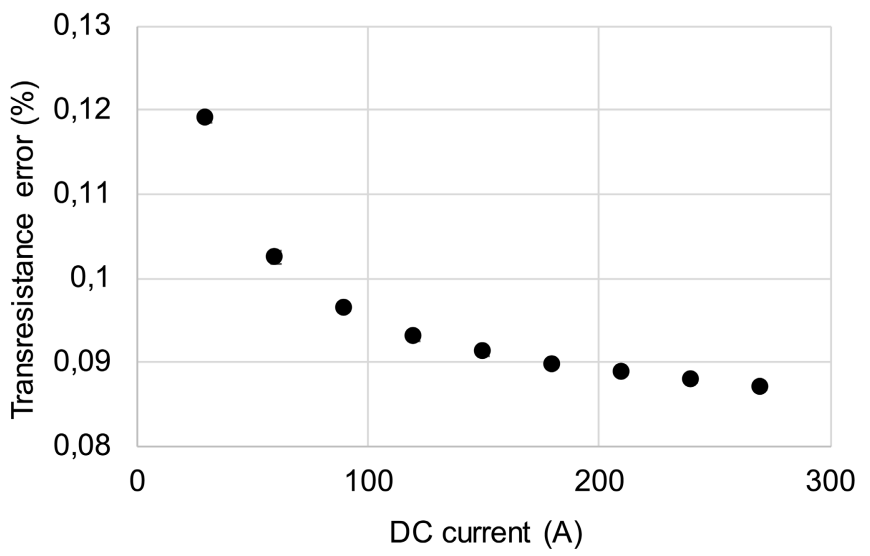

Fig. 5. Measured transresistance error of the current shunt under test as a function of applied dc current for the chopped waveform described in the text.

corresponding to another $0.03 \%$ for a realistic temperature range between $-20{ }^{\circ} \mathrm{C}$ and $+40{ }^{\circ} \mathrm{C}$.

In a separate measurement, the current dependence caused by intrinsic properties of the shunt other than dissipation has been determined. To do so, the electronic load is programmed with a chopped current profile in which the current is alternately switched ON for $5 \mathrm{~s}$ and switched OFF for $10 \mathrm{~s}$. The current level is increased by $30 \mathrm{~A}$ with each step to a maximum of $270 \mathrm{~A}$. The whole sequence is repeated five times, with several minutes in between. From the results, shown in Fig. 5, it can be seen that the transresistance error increases with decreasing current. The largest error deviates by $0.03 \%$ from the smallest error measured at the largest current, which resembles the value obtained before the heating sets in (as can be read from Fig. 4). The standard deviation of the measurements, shown as error bars in the figure, is typically a few parts per million only, which is not noticeable in the figure.

To mimic realistic operating conditions, a waveform is used that resembles the power consumption of a real traction unit. For this measurement, we used current data recorded at the pantograph of a traction unit traveling between successive underground stations of Metro de Madrid. The corresponding loading patterns are programmed into the memory of the

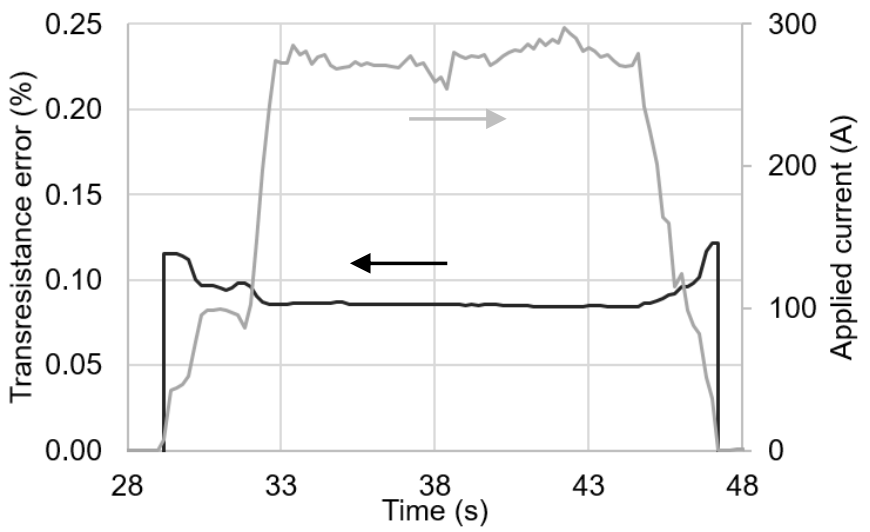

Fig. 6. Measured transresistance error of the current shunt under test (black line, left axis) during a simulated segment of a trip of an underground traction unit with the corresponding dc current applied (gray line, right axis).

electronic load. Both sensors can follow the dynamic behavior of the current. As expected, Fig. 6 reveals that the measurements resemble the behavior observed in Fig. 5, that is, the error increases with decreasing current. The onset of the heating effect can also be observed between 33 and $43 \mathrm{~s}$, in which the applied current is approximately constant, whereas the transresistance error shows a minor decrease. Hence, the effect of the dynamics of the current waveform is covered by the effect of dissipation and the intrinsic current dependence already investigated. Alternatively, one might argue that when calibrating the current shunt under realistic dynamic conditions, the effect of dissipation and intrinsic current dependence is included. Note that this observation can be considered a cross check of the ability of the reference sensor for correctly measuring dynamic de signals.

To determine the effect of ac distortion on the current shunt, the electronic load is programmed with de signals with and without additional ac components. From the measurements described in Section V, it was concluded that the sensor used as a reference in the revised measurement setup is not influenced by ac distortions to within a few parts in $10^{6}$. From the measurements described here, it was found that, within the error bars representing the standard deviation of the measurements of a few parts in $10^{6}$, the current shunt under test shows no dependence on the frequency or amplitude of the ac distortion either.

The total uncertainty of the transresistance ratio of the current shunt under test when used under realistic conditions is determined by the reference sensor (Table I), dissipation, intrinsic current dependence, the influence of ac distortion, the accuracy of the voltmeter ratio, the temperature dependence, and the standard deviation of the measurements (type A uncertainty). The total uncertainty budget for a dc input current of $270 \mathrm{~A}$ is shown in Table II, and the total expanded uncertainty is $0.05 \%(k=2)$. For dc currents down to $30 \mathrm{~A}$, the total uncertainty will be similar. Note that if this current shunt is used in real railway applications, another $0.03 \%$ (rectangular distribution) needs to be added due to its temperature coefficient of resistance. 
TABLE II

UnCERTAinty Budget of The CURREnt Shunt Under TESt AT 270 A DC WHEN UsEd Under Distorted CONDITIONS

\begin{tabular}{lccc}
\hline $\begin{array}{l}\text { Uncertainty } \\
\text { component }\end{array}$ & $\begin{array}{c}\text { Uncertainty } \\
\text { contribution } \\
(\%)\end{array}$ & $\begin{array}{c}\mathrm{k}- \\
\text { factor }\end{array}$ & $\begin{array}{c}\text { Standard } \\
\text { uncertainty } \\
(\%)\end{array}$ \\
\hline \hline Reference sensor & 0.001 & 2 & 0.001 \\
Dissipation & 0.030 & $\sqrt{3}$ & 0.017 \\
Current dependence & 0.030 & $\sqrt{ } 3$ & 0.017 \\
AC distortion & 0.001 & $\sqrt{ } 3$ & 0.001 \\
Voltmeter ratio & 0.000 & $\sqrt{3}$ & 0.000 \\
Standard deviation & 0.001 & 1 & 0.001 \\
\hline Total $(\mathrm{k}=2)$ & 0.050 & 2 & 0.025 \\
\hline
\end{tabular}

\section{CONCLUSION}

A measurement setup has been realized to characterize the dc output error of current sensors under realistic operating conditions for railway applications. In the initial setup, suitable only for static signals and for sensors containing zero-flux current transducers, an ac distortion was applied through a separate winding. This setup was mainly used to prove that the reference sensor used in a revised version of the setup, based on a programmable electronic load, was immune to the ac distortion within a few parts in $10^{6}$. The total uncertainty of the reference sensor was found to be smaller than $10 \mu \Omega / \Omega(k=$ 2).

The revised setup employs a programmable electronic load, which makes it suitable to apply dynamic signals. The setup can deal with different types of current sensors or even complete current measurement systems. The current sensor under test is compared to a reference sensor, consisting of a highprecision zero-flux current transducer in combination with a broadband high-precision shunt resistor. Distorted operating conditions can be simulated for currents up to $600 \mathrm{~A}$.

For the specific sensor under test in this paper, a $100 \mu \Omega$ high-current shunt designed for measurements with currents up to $300 \mathrm{~A}$, the longer term effect of dissipative heating was found to be around $0.03 \%$ for an applied dc current of $270 \mathrm{~A}$. The short-term intrinsic current dependence was found to be around $0.03 \%$ as well when comparing the error at currents down to $30 \mathrm{~A}$ to the error determined at $270 \mathrm{~A}$. The effect of the ac distortion on the dc transresistance error was found to be within a few parts in $10^{6}$, which is negligible when compared to the other effects. The intrinsic current dependence and the onset of the heating effect were also observed when exposing the sensor to a dynamic current profile that was recorded during a trip between two successive underground train stations from Metro de Madrid. The total uncertainty of the reference sensor was found to be smaller than $0.05 \%$ $(k=2)$. However, it should be noted that the temperature in the laboratory was $23.0{ }^{\circ} \mathrm{C} \pm 0.5{ }^{\circ} \mathrm{C}$, and the shunt was equipped with a cooling fan, whereas in practical railway applications, the temperature can vary between, say, $-20{ }^{\circ} \mathrm{C}$ and $+40^{\circ} \mathrm{C}$, and active cooling of the shunt might be absent or less effective.

The observed transresistance error of the high-current shunt investigated in this paper was around $0.10 \%$. The accuracy of the total EMF of railway traction units, including current and voltage measurement and energy calculation, needs to be on the order of $0.2 \%-1 \%$ [3]. Hence, when taking the uncertainty into account as well, the shunt under investigation would be just satisfactory for most metering applications on-board trains.

The effect of ac distortion on the error of the shunt is shown to be negligible. It should be noted, however, that ac ripple might still influence the complete current measurement system. Modulation and spectral leakage might disturb the reading of the meter if it is not correctly configured. However, in this paper, the focus is on current sensors only, and the investigation of complete current measuring systems is left for future work.

From the results presented here, we conclude that the setup described in this paper is very useful for characterizing dc current sensors for practical railway applications under realistic distortion conditions. On the other hand, in this paper, the emphasis is on traceability of the measurements, including uncertainty analysis; details on the use of the setup for practical applications are described more extensively elsewhere [14]. Future work will concentrate on even more demanding current signals, such as chopped signals, and on the characterization of other types of transducers and complete current measurement systems.

\section{ACKNOWLEDGMENT}

The authors would like to thank J. Q. Fernández and F. Balic for providing their current profiles measured in the underground traction unit of Metro de Madrid and for their valuable discussions.

\section{REFERENCES}

[1] A.González-Gil, R. Palacin, P. Batty, and J. P. Powell, "A systems approach to reduce urban rail energy consumption," Energy Convers. Manage., vol. 80, pp. 509-524, Apr. 2014.

[2] Technical Specifications for Interoperability Relating to the Energy Subsystem of the Rail System in the Union, document 1301, Commission Regulation (EU), Nov. 2014.

[3] Railway Applications-Energy Measurement on Board Trains-Part 2: Energy Measuring, document EN 50463-2, 2012.

[4] A. Cataliotti, D. Di Cara, A. E. Emanuel, and S. Nuccio, "A novel approach to current transformer characterization in the presence of harmonic distortion," IEEE Trans. Instrum. Meas., vol. 58, no. 5, pp. 1446-1453, May 2009.

[5] A. P. J. van Deursen, P. A. A. F. Wouters, H. W. M. Smulders, and J. B. M. van Waes, "EMC measurements in railway power systems," in Proc. Int. Symp. Electromagn. Compat. (EMC Europe), Rome, Italy, Sep. 2012, pp. 1-6.

[6] G. Crotti, D. Giordano, A. D. Ferninc, D. Gallo, C. Landi, and M. Luiso, "A testbed for static and dynamic characterization of DC voltage and current transducers," IEEE 9th Int. Workshop Appl. Meas. Power Syst., Bologna, Italy, Sep. 2018, pp. 1-6.

[7] G. Crotti et al., "Pantograph-to-OHL arc: Conducted effects in dc railway supply system," in Proc. IEEE 9th Int. Workshop Appl. Meas. Power Syst. (AMPS), Bologna, Italy, Sep. 2018, pp. 1-6.

[8] H. E. van den Brom, G. Rietveld, and E. So, "Sampling current ratio measurement system for calibration of current transducers up to $10 \mathrm{kA}$ with $5 \cdot 10^{-6}$ uncertainty," IEEE Trans. Instrum. Meas, vol. 64, no. 6, pp. 1685-1691, Jun. 2015.

[9] G. Rietveld, J. H. N. van der Beek, and E. Houtzager, "Accurate DC current ratio measurements for primary currents up to $600 \mathrm{~A}$," IEEE Trans. Instrum. Meas., vol. 64, no. 11, pp. 3055-3061, Nov. 2015. 
[10] H. E. van den Brom, R. van Leeuwen, and R. Hornecker, "Characterization of a reference DC current sensor with AC distortion for railway applications," in Conf. Precis. Elect. Meas. Dig., Paris, France, Nov. 2018, pp. 1-6.

[11] H. C. Appelo, M. Groenenboom, and J. Lisser, "The zero-flux dc current transformer a high precision bipolar wide-band measuring device," IEEE Trans. Nucl. Sci., vol. NS-24, no. 3, pp. 1810-1811, Jun. 1977.

[12] W. G. K. Ihlenfeld and M. Seckelmann, "Simple algorithm for sampling synchronization of ADCs," IEEE Trans. Instrum. Meas., vol. 58, no. 4, pp. 781-785, Apr. 2009.

[13] K. Lind, T. Sørsdal, and H. Slinde, "Design, modeling, and verification of high-performance AC-DC current shunts from inexpensive components," IEEE Trans. Instrum. Meas., vol. 57, no. 1, pp. 176-181, Jan. 2008.

[14] H. E. van den Brom, R. van Leeuwen, and R. Hornecker, "Characterization of DC current sensors under distorted conditions for railway applications," in ESARS-ITEC Conf. Dig., Nottingham, U.K., Nov. 2018, pp. 1-6.

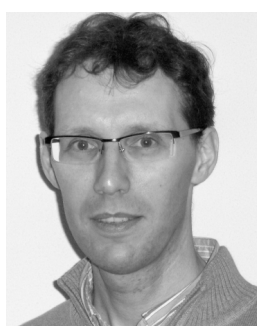

Helko E. van den Brom (M'14-SM'15) was born in Utrecht, The Netherlands, in 1971. He received the M.Sc. degree in theoretical solid-state physics from Utrecht University, Utrecht, in 1995 and the $\mathrm{Ph} . \mathrm{D}$. degree in experimental solid-state physics from Leiden University, Leiden, The Netherlands, in 2000 .

In 2000, he joined VSL, Delft, The Netherlands, where he was involved in the development of Josephson and SET-based electrical quantum standards and currently a Principle Scientist. His current research interests include power quality, high-current transducers, sampling systems, and ac Josephson voltage standards.

Dr. van den Brom is a Member of the Dutch Physical Society, a Technical Assessor for the Dutch Accreditation Council, a contact person for VSL in the technical committee of electricity and magnetism, and the subcommittee of dc and quantum electrical measurements of the European Association of National Metrology Institutes. He was a recipient of the Best Ph.D. Paper Award of the Dutch Journal of Physics in 2000.

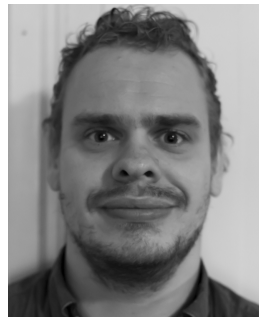

Ronald van Leeuwen was born in Nieuwerkerk aan den Ijssel, The Netherlands. He received the M.Sc. and Ph.D. degrees in applied physics from the Delft University of Technology, Delft, The Netherlands, in 2010 and 2015, respectively.

In 2017, he joined VSL, Delft, The Netherlands, where he was involved in the development of measurement techniques for high-current transducers and new techniques for testing of static electricity meters.

Dr. van Leeuwen was a recipient of the 2014 NEVAC Prize (Dutch vacuum society) for the best scientific article Luisteren naar nanodrums met atomaire dikte.

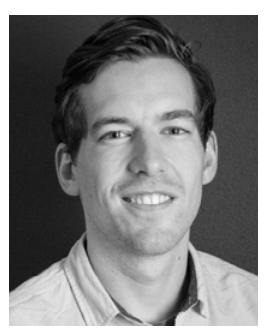

Ralph Hornecker was born in Endingen, Germany, in 1988. He received the B.Eng. degree in electronics from the University of Applied Sciences Aschaffenburg, Aschaffenburg, Germany, in 2017.

In 2017, he joined VSL, Delft, The Netherlands, where he is involved in power quality, low-frequency high-current measurement, and the development of a quantum alternating-current voltage standard. 